\title{
A RARE CASE OF STARGARDT'S DISEASE
}

\author{
E. Mermeklieva' ${ }^{1}$ K. Kamenarova ${ }^{2}$ \\ ${ }^{1}$ Clinic of Ophthalmology, „Lozenetz“ University Hospital, Medical Faculty, \\ University „Sv. Kliment Ohridski“ -Sofia, Bulgaria \\ ${ }^{2}$ Center of Molecular Medicine, Department of Medical Chemistry and Biochemistry, \\ Medical University - Sofia, Bulgaria
}

\begin{abstract}
Objective: To describe a clinical case of rare eye diseases - Stargardt's disease. Material and methods: A detailed clinical examination, fundus autofluorescence, optical coherence tomography and electrophysiological studies were performed. The clinical diagnosis was also genetically confirmed. Results: A classic Stargardt's disease phenotype was found in a 10-year old boy with decreased visual acuity, atrophy of the photoreceptors and retinal pigment epithelium layers in the macula, plus hypoautofluorescence in the fovea. In full-field ERG there was no diffuse cone involvement. Multifocal ERG demonstrated a lower cone activity in the area of the central macula in both eyes, which is characteristic for hereditary maculopathies and differentiates them from cone-rod dystrophies, in which generalized damage of the photoreceptors in the retina may be observed. The genetic studies identified two missense mutations: c.3113C> T (p.Ala1038Val) and c.1622T> $C$ (p.Leu541Pro) in a cis-position and a missense mutation c.2588G > C (p.Gly863Ala) in the other allele of ABCA4 gene. The two pathogenic variants $c .3113 C>T$ and $c .1622 T>$ C formed a complex allele p. [A1038V; L541P], which was found in the genome of the asymptomatic mother. The other mutation c.2588G> C affects a highly conserved amino acid from the ABCA4 protein (p.Gly863Ala) and was inherited from the patient's clinically healthy father, who was a heterozygous carrier. Conclusion: The comprehensive clinical, electrophysiological and genetic testing of patients with rare hereditary retinal dystrophies is essential for the correct diagnosis and the choice of therapeutic behavior.
\end{abstract}

Key words: hereditary retinal dystrophy, Stargardt's disease, electrophysiology, genetics

Corresponding author: Assoc. Prof. Elena Mermeklieva, Clinic of Ophthalmology, "Lozenetz" University Hospital, Medical Faculty, Sofia University "Sv. Kliment Ohridski" Sofia, 1 "Koziak" Street, Sofia, Bulgaria, e-mail: elenamermeklieva@yahoo.com

RECEIVED: 5 October 2020; ACCEPTED: 20 November 2020

\section{INTRODUCTION}

T he "Rare eye diseases" occur with a relatively low frequency in the human population. The hereditary retinal dystrophies consist the largest part of them. Stargardt's disease is the most common juvenile macular dystrophy and a common cause of central vision loss before the age of
50. The first manifestation occurs at different ages even among individuals within the same family. Most patients retain fair visual acuity $(0.2-0.3)$. Central visual field defects may be observed. The disease is a slowly progressive one. Its prevalence is 1:8000 population [1, 2]. The inheritance is usually autosomal recessive (AR) and is due to mutations in ABCA4 gene, which encodes the synthesis of a transporter protein, 
expressed in the outer segments of the retinal rods. The role of this protein is to support the transport of vitamin $\mathrm{A}$ (necessary for the formation of photosensitive pigments in the retinal rods and cones) from the photoreceptors back to the retinal pigment epithelial (RPE) cells, where the vitamin A molecules are recycled and re-used. In Stargardt's disease, this protein is either absent or has reduced function, causing accumulation of vitamin A dimers in RPE cells as well as in the photoreceptor cells thus damaging them. The gene is a very large one (50 exons) and is characterized by high polymorphism. To date, more than 900 variants of mutations of this gene have been identified. Other mutations that elicit the same phenotype are: STGD4, ELOVL4, as well as mutations in $\mathrm{PRPH} 2$ and PROM1 genes [3, 4].

The classic phenotype of Stargardt's disease is characterized by atrophy of the fovea, surrounded by discrete, yellowish, round flecks at the level of RPE, all starting in adolescence. If the flecks are scattered all over the fundus, the condition is called fundus flavimaculatus [1].

Characteristic changes are found on autofluorescence of the fundus (FAF): peripapillary atrophy of RPE, hypoautofluorescence of the fovea, and radially outward expanding pattern of hyper-fluorescent flecks. The clinical diagnosis of Stargardt's disease can be confirmed by the presence of the so-called "choroid silence" on fluorescein angiography (FA), due to the underlying choroidal fluorescence masked by the extensive area of abnormal RPE. Areas of hypo- and hyperfluorescence are observed in the macula. As the disease progresses, lipofuscin-like material accumulates in RPE $[5,6]$.
In ERG the photopic response is normal or subnormal, the scotopic response is usually normal. Electrooculography (EOG) is usually subnormal. The differential diagnosis includes all conditions that lead to the picture of "bull's eye" - the different cone- and cone-rod dystrophies.

\section{CASE DESCRIPTION}

A 10-year old boy had a detailed clinical examination, FAF, FA, optical coherence tomography (OCT), visual field testing, electrophysiological (EF) tests - full-field ERG (ffERG) and multifocal ERG (mfERG). The clinical diagnosis was suspected, and genetically confirmed, using the MiSeq platform (Illumina $\circledast$ ) and the TruSight One (Illumina $($ ) gene panel for sequencing 4813 genes associated with all inherited diseases. The study met the criteria of ethical standards for good medical practice. The patient provided informed consent in compliance with the ethics standards and the Helsinki Declaration (2013).

The patient's complaints included a gradual decrease of vision with one year duration. The parents did not report a family history. The best corrected visual acuity of the right eye (RE) was $B C V A=0.15$ and of the left eye (LE) $B C V A=0.3$. Pigment displacement in the central macula was observed in the fundus, thus creating a granular appearance of the macula. Hypoautofluorescence in the fovea was found on FAF due to the abnormal RPE. The characteristic "choroid silence" and granular hyperfluorescence of the macula were demonstrated by FA. Atrophy of the photoreceptors and RPE layers and accumulation of hyperreflective material in the macular area were visualized in OCT (Fig. 1, 2 and 3).
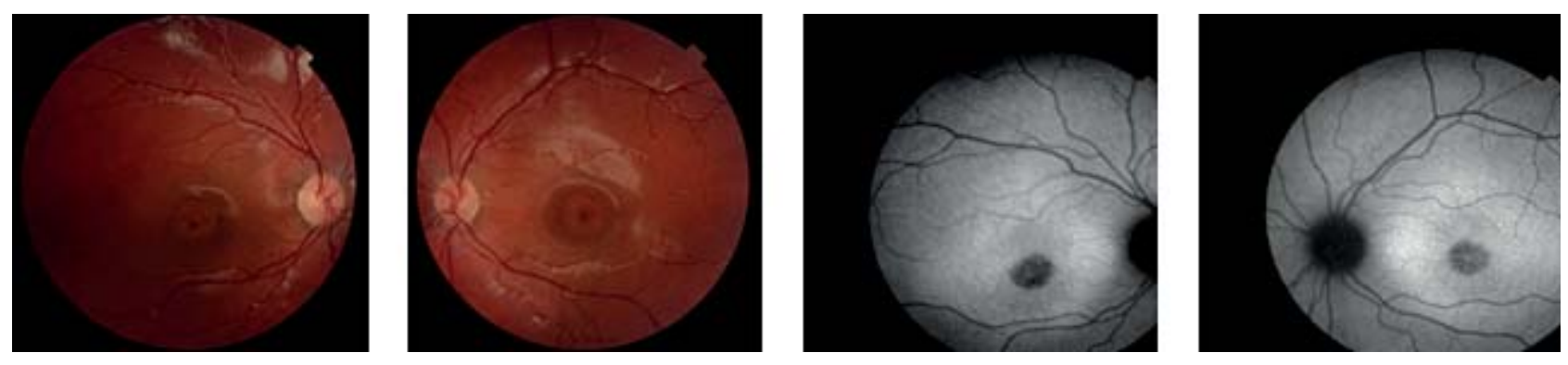

Fig. 1. Fundus photography and FAF of the patient (RE and LE)
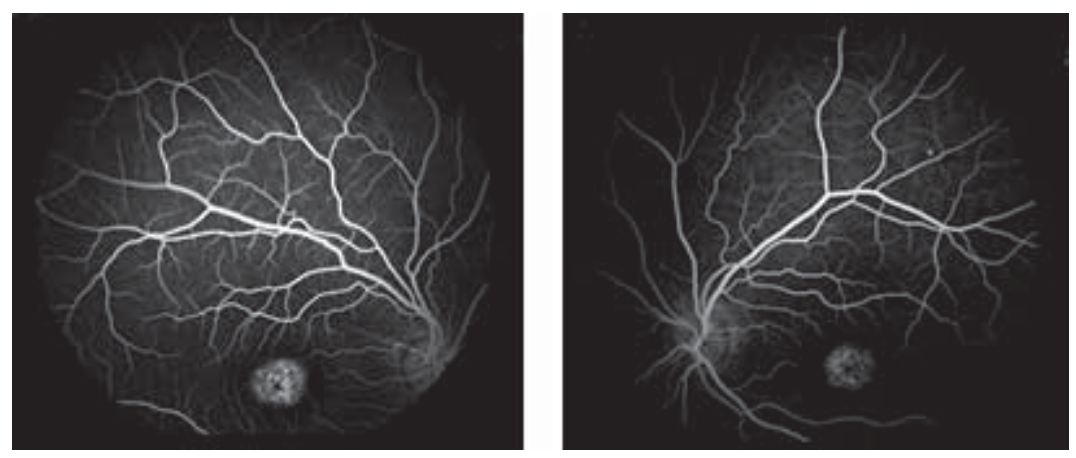

Fig. 2. FA of the patient (RE and LE) 

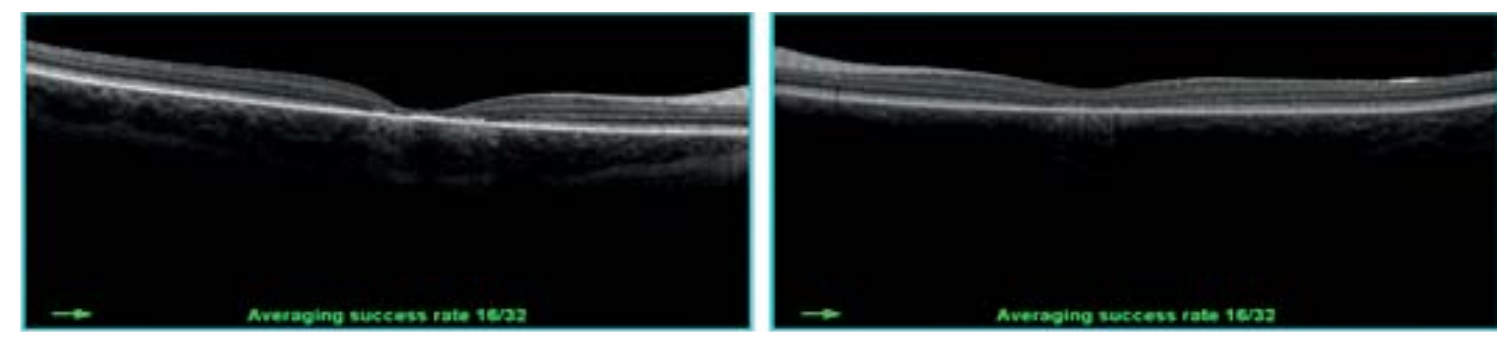

Fig. 3. OCT of the patient (RE and LE)

A central scotoma was found on computer perimetry (Fig. 4).

FfERG, which measures the generalized activity of rods and cones, demonstrated no diffuse cones involvement, as their EF response was quite preserved, with slightly lower amplitude than that of he rods, which were normal, and more affected in RE. However, mfERG, which can assess localized macular damage, demonstrated a lower cone activity in the area of the central macula in both eyes (Fig. 5 and Fig. 6).
Involvement of cone function was found only in the area of the central macula, a finding characteristic for hereditary maculopathies. This differentiates them from cone-rod dystrophies, which themselves are characterized by generalized damage of the photoreceptors in the retina.

The genetic studies identified two missense mutations in the proband: $c .3113 \mathrm{C}>\mathrm{T}$ (p.Ala1038Val) and c.1622T> C (p.Leu541Pro) in a cis-position and a missense mutation c.2588G $>C$ (p.Gly863Ala) in the
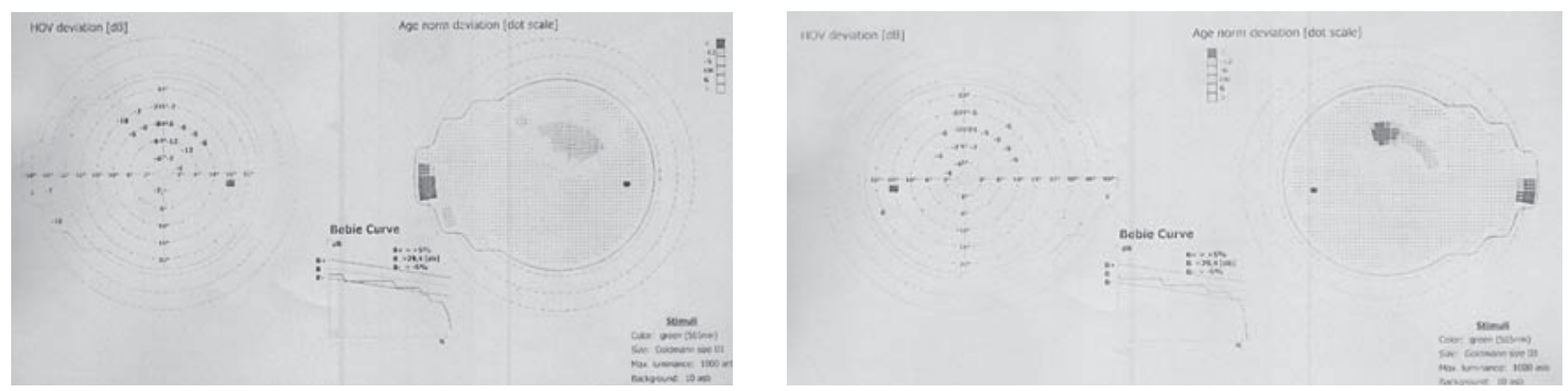

Fig. 4. Visual field testing of the patient (RE and LE)

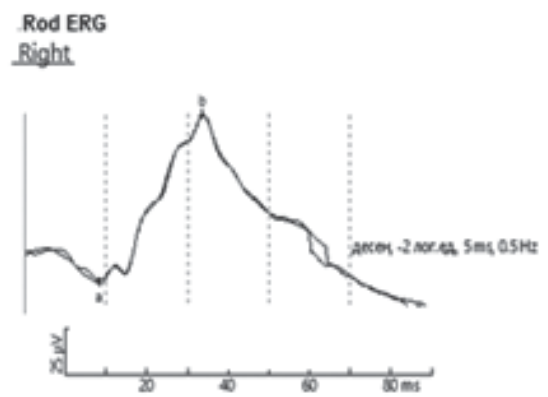

Rod ERG

Left.
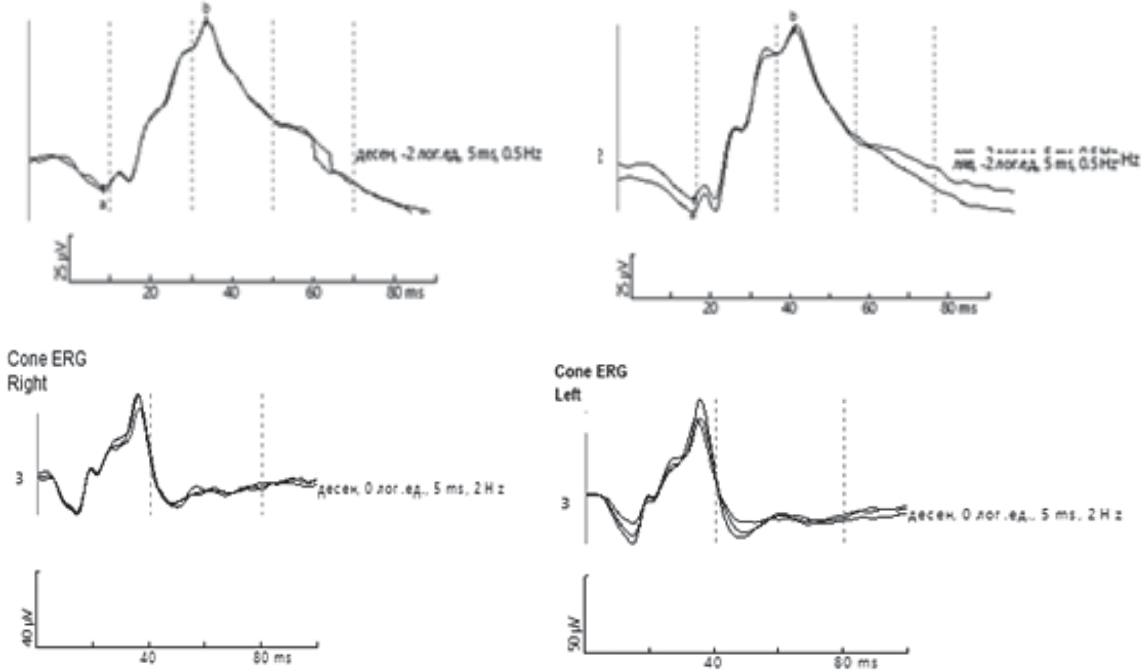

Fig. 5. FfERG of the patient 

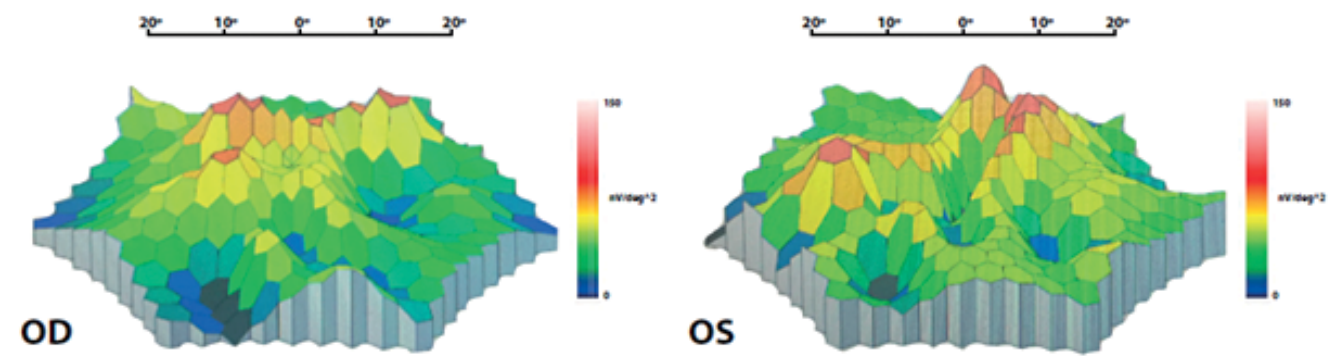

Fig. 6. MfERG of the patient (RE and LE)

other allele of ABCA4 gene. The two pathogenic variants c.3113C> T and c.1622T>C formed the complex allele p. (A1038V; L541P), which was found in the same copy of ABCA4 in the genome of the asymptonatic mother. The other mutation c.2588G> $C$ affects a highly conserved amino acid from the ABCA4 protein (p.Gly863Ala) and was inherited from the patient's clinically healthy father, who was a heterozygous carrier.

\section{DISCUSSION}

A classic Stargardt disease phenotype was found in a 10-year old boy with decreased visual acuity, atrophy of the photoreceptors and RPE layers in the macula, plus hypoautofluorescence in the fovea. EF tests, which are considered as the "golden standard" in diagnosis of hereditary retinal dystrophies, are very informative, because the appropriate EF test can made a differential diagnosis between the different types of hereditary retinal dystrophies $[5,8$, 9]. In this case, the ffERG test found that there was no diffuse cone involvement, characteristic for conerod dystrophies, while mfERG demonstrated lower cone activity in the central macula in both eyes, which is characteristic for hereditary maculopathies and differentiates them from cone-rod dystrophies, in which generalized damage of the photoreceptors in the retina is observed. The correct diagnosis is of great importance for the therapeutic algorithm. Vitamin A should be avoided in patients with Stargardt's disease as it may accelerate the accumulation of lipofuscin in the fovea. The correct diagnosis is also important for the application of new, still experimental therapies in these patients [11].

The complex alleles of ABCA4 gene are common and well studied from the very beginning of the research on this gene and its involvement in the pathogenesis of Stargardt's disease, as p.(A1038V; L541P) being one of the most frequent variants [1214]. The ABCA4-C.2588G> $C$ variant is present in population databases (ExAC $0.8 \%$ ), including mul- tiple homozygous individuals. Studies suggest that p.Gly863Ala is a mild variant that can lead to disease only when combined with a severe, pathogenic variant of ABCA4 (PMID: 10090887), such as p. (Leu541Pro; Ala1038Val) [14-16]. In combination, the two alleles p.(Ala1038Val; Leu541Pro) and p.Gly863Ala encoding two mutant copies of ABCA4 lead to Stargardt disease in the studied patient.

\section{CONCLUSION}

Comprehensive clinical, electrophysiological and genetic testing of patients with rare hereditary retinal dystrophies is essential for the correct diagnosis and the choice of therapeutic behavior.

\section{Declaration of conflicting interests}

The authors declared no potential conflicts of interest with respect to the research, authorship and/or publication of this article.

\section{Acknowledgments}

Supported by grants D-240/19.12.2019, Medical University of Sofia, Bulgaria, and KP-06-H33/12, NSF, MES, Bulgaria.

\section{REFERENCES}

1. AAO Retina and vitreous Basic and clinical science course 2017-2018, Course Book pp. 180-215.

2. Bowling B (ed.). Kanski's Clinical ophthalmology. A systematic approach 8th ed. Elsevier Limited, 2016, pp. 640-677.

3. Davis JL. The Blunt End: Surgical Challenges of Gene Therapy for Inherited Retinal Diseases. Am J Ophthalmol, 2018;196:xxv-xxix. doi: 10.1016/j.ajo.2018.08.038.

4. Rimoin DL., Connor JM, Pyeritz RE et al. Emery and Rimoin's Principles and Practice of Medical Genetics. 5th ed. Philadelphia: Churchill Livingstone; 2007, 137, 22-33.

5. Zahid S, Jayasundera T, Rhoades W et al. Clinical phenotypes and prognostic full-field electroretinographic findings in Stargardt disease. Am J Ophthalmol, 2013; 155(3): 465-473. 
6. Eagle RC, Lucier AC, Bernardino VB et al. Natural history of phenotypic changes in Stargardt macular dystrophy. Ophthalmic Genet, 2009; 30(2): 63-68.

7. Praidou $A$, Hagan $R$, Newman $W$ et al. Early diagnosis of Stargardt disease with multifocal electroretinogram in children. Int. Ophthalmol., 2014; 34(3): 613-621.

8. Vincent A, Robson AG, Holder GE. Pathognomonic (diagnostic) ERGs. A review and update. Retina. 2013; 33(1): 5-12.

9. Ajoy $\mathrm{V}$, Robson AG, Holder GE. Pathognomonic (Diagnostic) ERGs a review and update. Retina, 2013; 33(1): 5-12.

10. Creel DJ. The Electroretinogram and Electro-oculogram: Clinical Applications, Webvision, the organization of the retina and visual system, http://webvision.med.utah.edu/ book/electrophysiology/the-electroretinogram-clinical-pplications/

11. Sears AE, Bernstein PS, Cideciyan AV, et al. Towards Treatment of Stargardt Disease: Workshop Organized and Sponsored by the Foundation Fighting Blindness. TransI Vis Sci Technol, 2017, 6(5), 6. doi:10.1167/tvst.6.5.6
12. Salles MV, Motta FL, Martin R, et al. Variants in the ABCA4 gene in a Brazilian population with Stargardt disease. Mol Vis. 2018; 24: 546-559.

13. Tracewska AM, Kocyła-Karczmarewicz B, Rafalska A, et al. Genetic Spectrum of ABCA4-Associated Retinal Degeneration in Poland. Genes (Basel). 2019, 10(12), 959.

14. Khan M, Cornelis SS, Pozo-Valero MD, et al. Resolving the dark matter of ABCA4 for 1054 Stargardt disease probands through integrated genomics and transcriptomics. Genet Med. 2020; 22(7): 1235-1246.

15. Maugeri A, van Driel MA, van de Pol DJ, et al. The 2588G$\rightarrow C$ mutation in the ABCR gene is a mild frequent founder mutation in the Western European population and allows the classification of $A B C R$ mutations in patients with Stargardt disease. Am J Hum Genet. 1999; 64(4): 1024-1035.

16. Kamakari S, Kokkinou V, Koutsodontis G, et al. Corrigendum to "Mutation Spectrum of the ABCA4 Gene in a Greek Cohort with Stargardt Disease: Identification of Novel Mutations and Evidence of Three Prevalent Mutated Alleles". J Ophthalmol. 2018, 3039672. 